\title{
Effect of Dynamic Mechanical Compression on Actin Cytoskeleton Network of Human Mesenchymal Stem Cells (hMSCs) in Three Dimensional Collagen Constructs
}

\author{
Nicky F.C. Ho, Dr. Barbara P. Chan \\ Tissue Engineering Lab, Mechanical Engineering, \\ The University of Hong Kong, \\ Hong Kong \\ bpchan@hkucc.hku.hk
}

\begin{abstract}
Actin filament, one type of cytoskeletons, plays a central role in mediating cellular in responses to mechanical loading. Many mechanorgulation studies are restricted to 2D models using isolated cells or monolayer cultures, even though it is know that cells behave differently in term of cell morphology, cell matrix adhesion composition and matrix mediated force transmission when they are in 3D configuration. This current study investigates the temporal change of actin network of hMSCs entrapped in 3D collagen construct upon cyclic compression. Human bone marrow mesenchymal stem cells were encapsulated in cylindrical collagen construct. A micromanipulator based loading device coupled to fluorescent microscope was used to deliver compression loading to the construct with $10 \%$ strain at $1 \mathrm{~Hz}$ for different period of time. Rhodamine phalloidin was used to stain for the actin filament network to hMSC in the construct at different time points postcompression. An optimized loading protocol with $5 \mathrm{hrs}$ of continuous loading was delivered. Actin network concentrated at the cell periphery of cells exhibiting round morphology was observed immediately while elongated and polarized actin network was found after $\mathbf{2 4}$ hours . Detailed characterization of actin filament organization and their association with cell-matrix interaction molecules are warrented before the mechanisms of compression-induced hMSC alignment can be delineated
\end{abstract}

Keywords-component; Mechanoregulation, Actin filament, compression, human mesenchymal stem cells

\section{INTRODUCTION}

Actin filaments, together with microtubules and intermediate filaments constitute the three types of cytoskeleton and forming a tensegrity structure [1]-[2], playing central roles in cellular mechanoregulation. Actin filaments can participate in different cellular events, such as cell migration, proliferation, morphological changes and differentiation. They can reorganize in response to mechanical loading [3]. Reorganization of the actin filament network depends on the balance between the polymerization and depolymerization of actin filament with its monomer G-actin and the participation of actin binding proteins such as Arp2/3 [4] and actin depolymerization proteins such as cofilin [5]. Actin filaments, together with actin binding proteins, are important for different cellular events.

Many mechanoregulation studies are restricted to 2D models using single cells or monolayer cultures. However, in native tissue, cells are residing in three dimensional extracellular matrix. Cells behave differently when they are cultured on two dimensional monolayer or when cultured within three dimensional extra-cellular matrix [6]. For example, fibroblasts can realign perpendicular to the loading direction in 2D model [7] whereas they will realign parallel to the loading direction in 3D models [8]. Transmission of force in $2 \mathrm{D}$ culture is also different that in 3D models. These suggest that results of $2 \mathrm{D}$ cultures may not be translatable to the 3D models.

Mechanoregulation is the study of "how cell senses mechanical signals and transduces into cascade biochemical signals ultimately leading to a host of biological response" [9]. Our previous study has demonstrated that hMSCs culture in three dimensional type I collagen construct can respond to cyclic compressive loading and realign parallel to the loading direction after 7 days compression [ref]. In order to further investigate the mechanism of such compression-induced hMSCs alignment response, the aim of this study is to investigate the temporal changes of actin network of hMSCs under compressive loading. This study contributes to designing bioreactor loading protocol of hMSCs based functional tissue engineering.

\section{METHOD}

\section{A. Fabricatoion of Three dimensional hMSCs collagen construct}

Procedures for fabricating the 3D hMSCs collagen construct were described previously $[10,11]$. Briefly, rat tail type I collagen solution (BD Biosciences) was neutralized with sodium hydroxide (Sigma-Aldrich) and mixed with hMSCs suspended in culture medium (DMEM-LG, supplemented with $10 \%$ FBS and $100 \mathrm{U} / \mathrm{mL}$ penicillin and $100 \mathrm{ug} / \mathrm{mL}$ streptomycin and $2 \mathrm{mM}$ glutaMax) at a final collagen concentration of $2 \mathrm{mg} / \mathrm{ml}$ with a final cell density $1 \times 10^{6}$ cell $/ \mathrm{ml}$. $30 \mathrm{ul}$ of hMSCs collagen aliquots were transferred to a custom made cylindrical chamber and incubate for $60 \mathrm{mins}$ for gelation. The construct were cultured as free suspension state for 4 days before compression. 


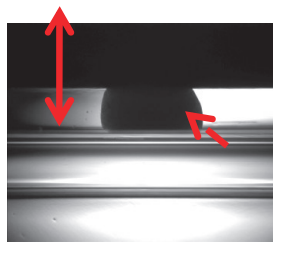

Fig. 1 Captured image during compression. Double arrow: compression direction. Single arrow: hMSCs-collagen constructs

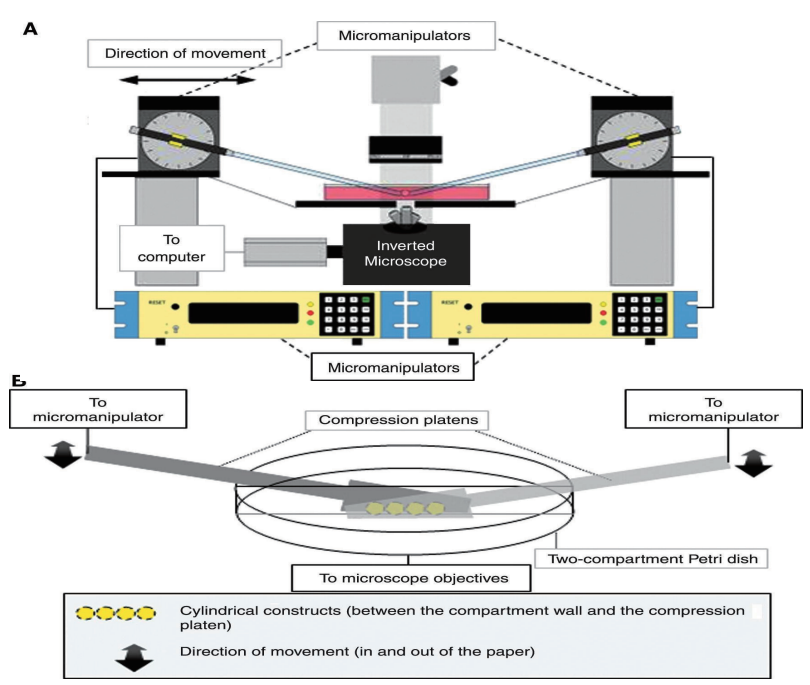

Fig. 2 The compression system. 2A: The diagram of micromanipulator based loading system, including a pair of micromanipulator. 2B: The loading set up. A pair of custom made platens is connected to the micromanipulator and delivers dynamic compression to the construct. [2]

\section{B. Optimization of loading protocol}

Cyclic compression was performed on constructs through using the custom-made micromanipulator-based loading system as illustrated in fig. 1 and fig. 2 [11]. Briefly, hMSCs Collagen constructs were transferred to a 2 compartment dish, which was placed onto a heatplate pre-warmed to $37^{\circ} \mathrm{C}$. Positions of the constructs were adjusted thickness of the constructs can be measured. The loading program was set with preloading at $10 \%$ strain and then compression loading for another $10 \%$ strain at frequency $1 \mathrm{~Hz}$. In order to further optimized our previously established consecutive loading protocol [9], continuous loading for 5 hours was examined. During the compression, medium was changed every 2 hours. The constructs were fixed immediately by $4 \%$ PFA, 12 hours and 24 hours after compression. The fixed constructs were rinsed by $1 x$ PBS to wash out excessive PFA and equilibrated with into $30 \%$ sucrose before preparing $15 \mu \mathrm{m}$ cryosections for fluorescent staining.

\section{Fluorescent staining of actin filament}

Briefly, sections were first washed in 1x PBS thrice and incubated by $0.1 \%$ Triton $\mathrm{X}-100$ for $5 \mathrm{mins}$. Non-specific binding was blocked by $1 \%$ BSA (Sigma-Aldrich Co.) for 30mins. Rhodamine Phalloidin (Molecular Probes, Inivitrogen) at a concentration of $165 \mathrm{nM}$ was used to stain the actin filament for 40mins and DAPI (Molecular Probes, Invitrogen) was used to counterstain the nuclei. Excessive dyes were washed with $1 \mathrm{X}$ PBS thrice before mounting the section by fluorescent mount medium (DAKO). All images were obtained by an inverted microscope (EclipseTE2000-U) equipped with Epi-Fluorescent unit (Nikon).

\section{Live and dead staining}

hMSCs collagen construct were washed in 1x PBS thrice and followed by incubating with $2 \mu \mathrm{M}$ Calcein $\mathrm{AM}$ and $4 \mathrm{Mm}$ Ethidium homodimer-1 (Molecular Probes, Invitrogen) for $45 \mathrm{mins}$ to stain for the live and dead cells. Stained constructs were rinsed with $1 \mathrm{xPBs}$ thrice to remove excessive dye.

\section{RESULT}

\section{A. Temoral change of actin organization upon 5 hours compression}

Actin organzation in the cotnrol, without compression group was elongated, giving the cell bipolar shape with long extension as shown in fig. 3A. Immediately after 5 hours compression, actin organziation change from this elongated shaped to a smaller and round shape actin netowrk, resuting in in less cell extension (fig. 3B). After 12 hours post compression, although some of hMSC with round like actin organizations were observed, some of the actin structure has been recovered, leading to a less rounded, more polarized or stellate shape cell morphology (fig. 3C). After 24 hours post compression, the actin organizations were similar to 12 hours post compression group, but with a longer extension, giving a bipolar and polarized cell morphology similar to hMSCs in control group.

\section{B. Live and Dead staining}

As shown in fig. $6 \mathrm{~A}$ and $\mathrm{B}$, both the control group and the 5 hours compression group showed high cell viability. Although the staining were performed for the whole construct rather than sectioned slides, the large proportion of live cell (green color) compared to dead cells (red colored dot) suggest that prolonged and continuous for 5 hours would not compromise cell viability.

\section{DISCUSSION}

Before compression, the actin organization was elongated, giving bipolar cell morphology. Immediately after compression, actin organized to give round cell morphology. Actin filaments are bundles forming pre-tensioned stress fiber [12], which connecting between integrins anchoring onto the collagen fibril. These pre-tensioned stress fibers are important for the cell morphology. Breakdown of stress fibers by cytochalasin D drug treatment would cause collapse of the cell [12]. During compression, cells were deformed. The change of the actin organization and hMSCs morphology may be due to 

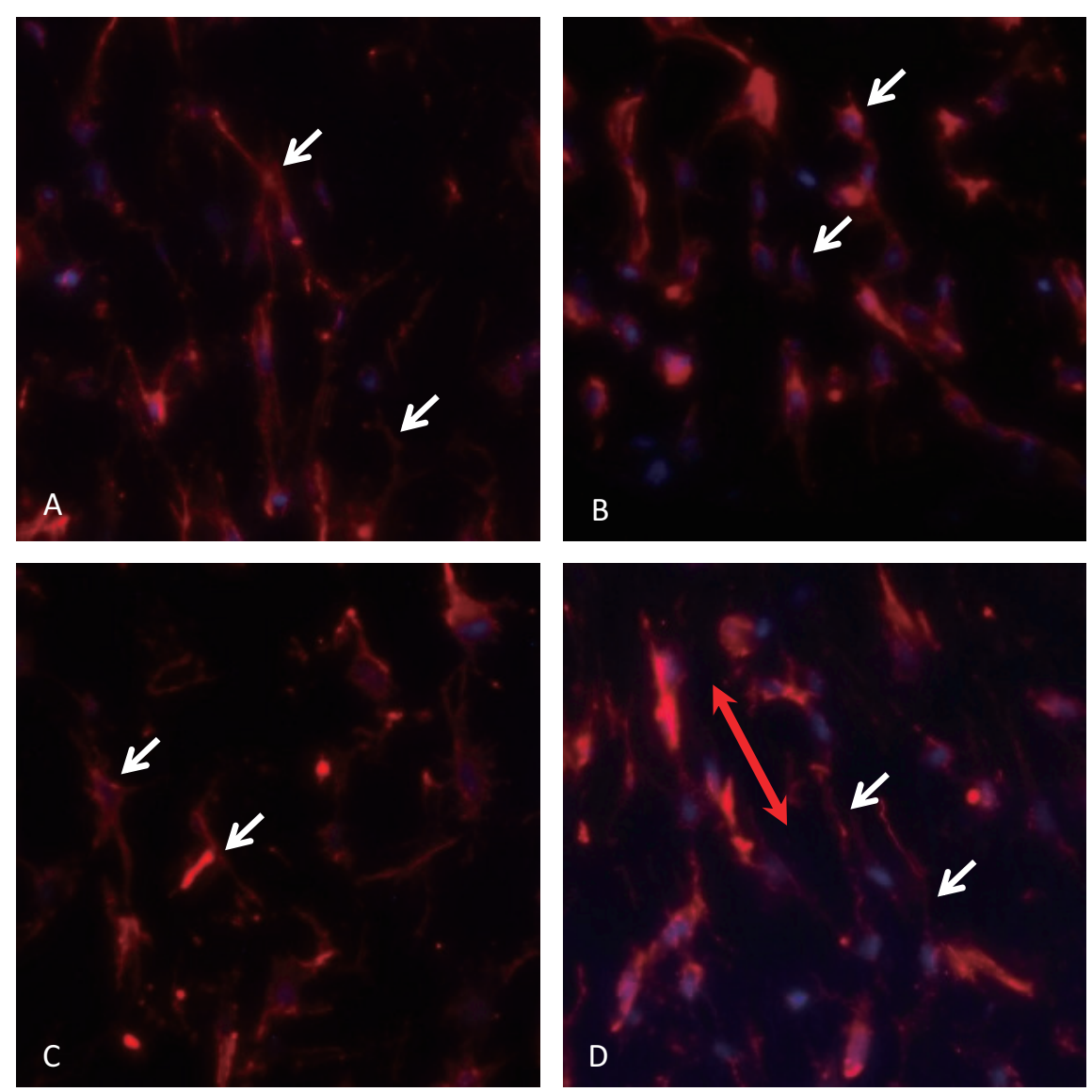

Fig. 3 Fluorescent image of actin staining. Actin filament (Red), Nuclei (Blue) A: Control without compression, with arrow indicated the polarized actin organization. B: Immediately after compression. Arrows indicated the round actin organization. C: 12 hours after compression. Arrows indicated elongated, stellate actin organization. D: 24 hours after compression. Arrows indicated the elongated, polarized actin organization. Double head arrow indicate the direction of compression
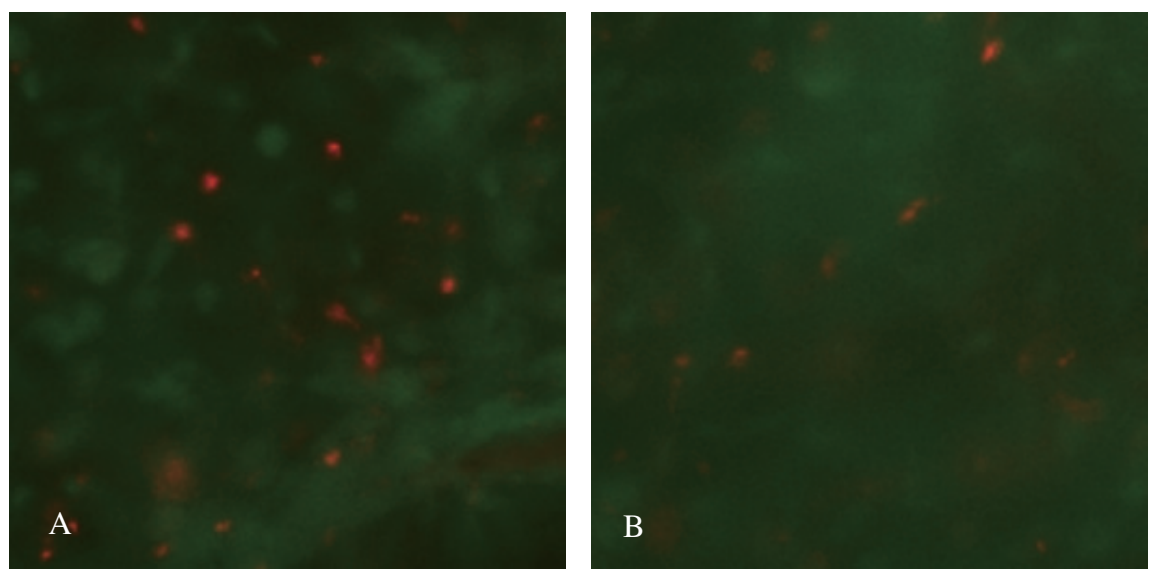

Fig. 4 Live and dead staining. Live cell (Green). Dead cell (Red). A: control, without compression. B: Immediately after 5 hours compression. $\mathrm{x} 100$.

the breakdown of the actin network because of the compressive loading. As observed in fig. 3B there are strong actin staining concentrate at the cell periphery. And after 24 hours, actin network recovered to a more polarized shape as seen in fig. 3D, with some of the hMSCs align parallel to the loading direction. These may suggested that the immediate effect of the compression on the actin dynamics and actin network will be kept modified even after loading. When cells culturing on monolayer migrated, nucleation of actin and branching of the actin filament were occurred at the cell lamellopodia which leaded to the push out of the cell membrane and thus the migration. In our model, the breakdown of the actin network and the highly concentrated actin at the cell periphery may indicated that the actin network is modified at this state, hMSCs was under morphological change or migration, which lead to the polarized shape as seen in 24 hours post compression group. 


\section{ACKNOWLEDGMENT}

THIS WORK WAS SUPPORTED BY GRANTS FROM AOSPINE (AOSBRC-07-06); THE INNOVATION AND TECHNOLOGY COMMISSION, THE HONG KONG GOVERNMENT (ITS-133-08) AND THE STRATEGIC RESEARCH THEMES OF THE UNIVERSITY OF HONG KONG ON BIOMEDICAL ENGINEERING AND BIONANOTECHNOLOGY.

\section{REFERENCES}

[1] D. E. Ingber, "Tensegrity I. Cell structure and hierarchical systems biology," J Cell Sci, vol. 116(Pt 7), pp. 1157-73, 2003

[2] D. E. Ingber, "Tensegrity II. How structural networks influence cellular information processing networks," J Cell Sci, vol. 116(Pt 8), pp. 1397408, 2008.

[3] F. M. Pavalko, N.X. Chen, C. H. Turner, D. B. Burr, S. Atkinson, Y. F. Hsieh, J. QiU, R. L. Duncan, "Fluid shear-induced mechanical signal in MC3T3-E1 osteoblasts requires cytoskeleton-integrin interactions, " $A M$ J Physiol, vol. 275 (6 Pt 1), pp. 1591-601, 1998.

[4] C. L. Clainche, M. F. Carlier, "Regulation of actin assembly associated with protrusion and adhesion in cell migration," Physiol. Rev, vol. 88, pp. 489-513, 2008.

[5] G. Karp, "The cytoskeleton and Cell motility," Cell and Molecular Biology. Concept and Experiments, $5^{\text {th }}$ ed. Wiley, 2007, pp. 328-377.

[6] E. Cukierman, "Taking cell-matrix adhesions to the third dimension," Science, vol. 294(5547), pp. 1708-12, 2001.

[7] C. Neidlinger-Wilke, E. Grood, L. Claes, R. Brand, "Fibroblast orientation to stretch begin within three hours," J orthop Res, vol. 19, pp. 286, 2001.

[8] D. R. Henshaw, E. Attia, M. Bhargava, J. A. Hannafin, "Canine ACL fibroblast integrin expression and cell alignment in response to cyclic tensile strain in three-dimensional collagen gels," J Orthop Res, vol. 24(3), pp. 481-90, 2006.

[9] M. R. K. Mofrad, R. F. Kamm, "Cytoskeleton mechanics models and measurements," Cambride, pp. 1, 2006.

[10] B. P. Chan, T. Y. Hui, C. W. Yeung, J. Li, I. Mo, G. C. F. Chan, "Selfassembled collagen-human mesenchymal stem cell microspheres for regenerative medicine," Biomaterials, vol. 28(31), pp. 4652-66, 2007.

[11] K. L. Au-Yeung, K. Y. Sze, M. H. Sham, B. P. Chan, "Development of a micromanipulator-based loading device for mechanoregulation study of human mesenchymal stem cells in three-dimensional collagen constructs,"Tissue Eng Part C Method, vol. 16(1), pp. 93-107, 2009.

[12] K. Naygayama, T. Matsumoto, "Contribution of actin filament and microtubules to quasi-in situ tensile properties and internal force balance of cultured smooth muscle cells on a substrate," Am J Physiol Cell Physiol, vol. 295(6), pp. 1569-78, 2008

[13] L. M. Machesky, S. J. Atkinson, C. Ampe, J. Vandekerckhove and T. D. Pollard, "Purification of a cortical complex containing 2 unconventional actins from acanthamoeba by affinity-chromatography on profilinagarose," J. Cell Biol. Vol. 127(1), pp. 107-115, 1994

[14] R. C. May, "The Arp2/3 complex: a central regulator of the actin cytoskeleton," Cell. Mo. Life. Sci, vol. 58(11). pp. 1607-26, 2001 